\title{
Automatic Segmentation of the Prostate from Ultrasound Data Using Feature-Based Self Organizing Map
}

\author{
Amjad Zaim \\ Amman University, Biomedical Engineering Department \\ 19328 Amman, Jordan \\ Zaim_amjad@yahoo.com
}

\begin{abstract}
Traditional segmentation methods cannot provide satisfying results for extraction of prostate gland from Transrectal Ultrasound (TRUS) images because of the presence of strong speckle noise and shadow artifacts. Most ultrasound image segmentation techniques that adopt model-based approach such as active contour are considered semi-automatic because they require initial seeds or contours to be manually identified. In this paper, we propose a method for automatic segmentation of prostate using feature-based self organizing map (SOM). Median filtering and top hat transform are first applied to remove speckle noise. A technique is developed to remove ultrasound-specific speckles using texturebased thresholding. An SOM algorithm is employed to identify prostate pixels taking spatial information, gray-level as well as texture information to form its input vector. The clustered image is then processed to produce a fully connected prostate contour. A number of experiments comparing extracted contours with manually-delineated contours validated the performance of our method.
\end{abstract}

\section{Introduction}

Prostate cancer is the most commonly diagnosed cancer in men and the second highest North American mortality rate among all cancers in men, surpassed only by lung cancer [1]. Almost all common methods of diagnoses and treatments, such as needlebiopsy and brachytherapy, respectively, rely on 2-D or 3-D ultrasound data to accurately locate the prostate gland and device an effective plan for therapy. Transrectal ultrasound utilizes a cylinder shape probe that images the prostate through the rectum and ultimately reconstruct a $3-\mathrm{D}$ model of the prostate $[1,2]$. TRUS is relatively inexpensive and easy to use compared to other imaging modalities such as MRI or CT. In addition, the safety associated with ultrasound allows for real time monitoring of the prostate gland and accounts for any anatomical displacement or deformation. However, there are several drawbacks of ultrasound compared to other imaging modalities. These include low signal to noise ratio especially in low-contrast regions, the inability to image through bone or air, speckles which arise from constructive-destructive interference of the reflected waves and other artifacts. As a result, most modern treatment planning systems require that the prostate boundaries are manually delineated by an experienced sonographer, which requires extensive labor time and comes at the 
expense of spatial resolution particularly when large number of $2 \mathrm{D}$ images are available. Many efforts have been aimed at devising automatic or semi-automatic algorithms that could segment the prostate boundaries from the ultrasound images accurately and effectively and with minimal human intervention. A 3D discrete active deformable model was built by researchers to outline the prostate using initial polygonal contours defined in a number of slices and using edge maps to drive the deformation model $[3,4]$. Others have developed an algorithm for detecting prostate edges as a visual guidance for the user to manually follow [5]. Statistical shape models have also been developed to segment and differentiate between the various shapes of prostates using prior knowledge of the prostate region in ultrasound images. Neural Network has also been utilized to recognize the prostate geometry from a database of prostate shapes. Gabor filtering was designed to extract prostate features and train a KSVM neural network [6]. Adaptive edge-detection methods were also employed [7]. While some of these studies have reported accurate segmentation results, most still require substantial degree of user-interaction either to generate initial contours or to accumulate large number of prostate contours of various shapes. The contribution of the proposed model comes from its ability to detect prostate boundaries with minimal human intervention. The proposed method first applies a set of noise-removal filters to reduce speckle effects. SOM neural network is then employed to cluster similar regions using the spatial information, gray-level as well as texture information to form its input vector. The clustered image is then processed to produce a fully connected prostate contour. A number of experiments comparing the extracted contours with manually-delineated contours validated the performance of our method.

\section{Segmentation Method}

Our segmentation scheme is divided into three major tasks applied sequentially. The first task is to reduce noise and speckles that typically exist in ultrasound images and usually interfere in the segmentation process. This is accomplished via a set of preprocessing morphological operations as well as texture-based thresholding. The second task is to classify image pixels into discrete finite sets of regions including a prostate region via a two-stage SOM. The segmentation process associated with the use of the SOM network employs spatial, texture and gray-level information. Finally, the segmented image is further processed by a set of image processing algorithms to remove holes and scattered regions in the segmented image.

\subsection{Noise Removal Filtering}

Median Filtering. Median filter is first applied to reduce impulsive noise. This nonlinear filtering modifies the gray-levels of the image while preserving the original information. The center pixel of a $5 \times 5$ window is substituted with the median value of all the pixels in the window.

Top-Hat Transform. Top-hat transform filter is applied by morphological opening the image by a flat-top hexagon structure element with a radius of 9 . Morphological 
operation is a well-known procedure in image processing. The opening of an image A is obtained by first eroding the image with a structure element $\mathrm{B}$, after which one performs a dilation on this eroded image with the same structure element $[8,9]$. Mathematically, opening of A by B is defined as:

$$
A \circ B=(A \Theta B) \oplus B
$$

This process removes peaks of image surfaces smaller than hexagon leaving slowly varying background. This gray-level variation is further removed by subtracting the opened image from the original image. The resulting image difference image contains little or no information in the regions of low-signal amplitude. The resulting image is shown in the right side of Figure 1.

Morphological Closing. To reduce the impact of low-contrast areas left by the previous process, we perform morphological closing of the image with a hexagonal structuring element of radius 6. Morphological closing fuses narrow breaks and long gulfs in binary and gray-scale images [9]. The Closing of an image A by structuring element $\mathrm{B}$, is simply the opposite of opening A by B. Closing here is therefore is defined as the dilation of A by B followed by erosion of the results by B or:

$$
A \bullet B=(A \oplus B) \Theta B
$$

The initial dilation process removes the dark details and brighten the image. The subsequent erosion process darkens the image without reintroducing the details removed by dilation. We used a hexagonal shape structuring element of 6-pixel radius. This radius was chosen to be small enough to follow the details of the prostate contour but large enough to bridge small gaps in the image (Figure 2; left).

Region-Growing Thresholding. Looking at the resulting image (Figure 2; left) indicates that the previous filtering technique was able to outline speckle pattern that is visible in the image as connected regions of slightly "banana-shaped" white lines. This type of noise is a result of the impact of sound beam on perpendicular surfaces in the ultrasound field. To identify these speckles, we search through the gray-levels of the image for the point where regions are more connected and hence, less gray-level transitions. Spatial gray level co-occurrence or GLCM estimates image properties related to second order statistics and is one of the most well-known texture features [10]. The contrast measure of GLCM is defined as follows: let $r$ be a window in the image and $A_{k l}$ number of pairs of adjacent pixels within $r$ with grey-values $k$ and $l$ respectively. We can define the contrast of $r$ as:

$$
\text { Contrast }=\frac{\sum_{k, l=0}^{255}(k-l)^{2} \mathrm{~A}_{k, l}}{\sum_{k, l=0}^{255} \mathrm{~A}_{k, l}}
$$

The contrast is a measure for how many grey-value transitions there are in the region under consideration; the more adjacent pixels with a big difference in grey-value there are in $r$, the higher the contrast is. We utilize this feature to search the gray-level 
space for the threshold that maximizes the contrast and thus reduces speckles without leaving scattered gaps in the prostate. For our purpose, a threshold of 45 was found to be optimal to remove this type of speckles.
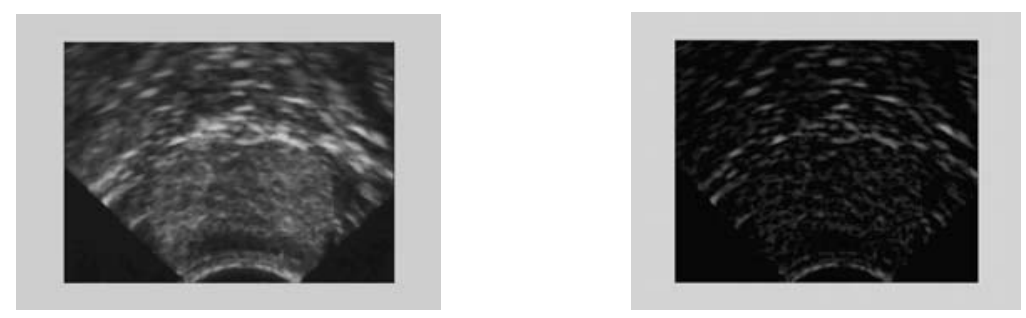

Fig. 1. Original TRUS image of the prostate (left). The image after applying morphological top-hat transform (right)
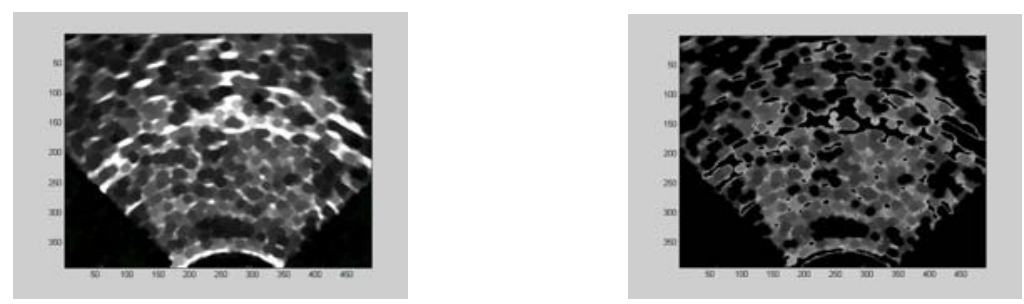

Fig. 2. Result of morphological closing (left). The same image after thresholding using GLCM contrast (right)

\subsection{Self-organizing Map}

SOM is considered an unsupervised neural network that can serve as a clustering tool for high-dimensional data [11]. It constructs a topology in which the highdimensional space is mapped onto map units in such a way that relative topology distances between input data are preserved. The map units usually form a twodimensional regular lattice. In our image every input unit is represented by a 4 dimensional feature vector. The features are center $x, y$ coordinates, the gray-scale level, as well as the contrast texture feature. The $x$ coordinate and the $y$-coordinate encode the spatial information of a pixel, and the gray-level value encode its intensity information. The fourth feature is a texture feature derived from an image block around the pixel of interest as calculated by Equation 1. Each four-dimensional feature vector is regarded as an input vector of the SOM network. The output of the SOM network is $n$ classes. The SOM network is trained as follows. Each map unit is associated with a reference vector. At first, all the reference vectors are randomly designated. Each input vector is compared with all the reference vectors and the unit whose reference vector is most similar to the input vector is identified. Then, the reference vectors 
neighboring to that of the identified unit are moved towards the input vector. Once training is accomplished, input data vectors that are topologically close are mapped to the same class.

Since we use Euclidean distance to measure the competition in SOM learning, it is necessary to normalize the input vectors, and weight features by importance. This prevent features of lesser importance from overriding more important features in the mappings. In this application, we found that the gray level intensities of the pixels are more important than other features to discriminate their classes. The size and the radius of the receptive fields of the SOM are adjustable by architecture parameters [11].

Our SOM consists of 2 layers. The first layer is a $2 \times 3$ network oriented in the 2D space. A total of 6 neurons allow Input data vectors that are topologically close to be mapped to the same class. That means the input vector space is divided into 6 classes. Each pixel is associated with a certain class after clustering. A second layer maps the 6 neurons from the first layer into a 2-class output. The two classes correspond to "prostate" and "non-prostate" classes. The first layer helps remove noise but retain textural features that are similar to prostate textures. However, pixels belong to the same class are not always connected. The second SOM layer refines the clustering operation by allowing clusters that are in close proximity to be identified and labeled. The spatial resolution was reduced 5 folds to accommodate the extensive computation of SOM. This is done by simply averaging every 5-pixel size window and assigning the mean value of the pixel intensity $I$ to the new image pixel $P$ such that:

$$
P_{i, j}=\frac{1}{25} \sum_{i}^{i+5} \sum_{j}^{j+5} I_{i, j}
$$

where $i=1,5, \ldots \mathrm{N}$ and $\mathrm{j}=1,5, \ldots, \mathrm{M}$ and the image size is $\mathrm{MxN}$. The result is a binary image with a map of the prostate with some scattered isolated points and holes within the contour image (Figure 3).

\subsection{Removal of Holes and Scattered Points}

The resulting binary image contains the prostate with holes within its contour as well as scattered regions outside its contour. We extract the prostate area by using morphological closing and opening operations in succession. These processes are commonly used to smooth, fill in, and/or remove objects in a grayscale or binary image[10]. As noted earlier, morphologic closing is equivalent to a dilation followed by an erosion with a structuring element. Similarly, morphologic opening is equivalent to an erosion followed by a dilation. The erosion stage of opening eliminates the scattered points outside the prostate. The dilation stage of closing fills the holes inside the prostate and restores boundary shapes. In our case, we used a disk-shaped structuring element with a radius of 6 pixels. Experiments have shown that this radius is large enough to fill most common size holes and hence yields satisfying results. As shown in figure 3, the resultant image contains a fully connected prostate region with all scatted points eliminated and all holes filled. 

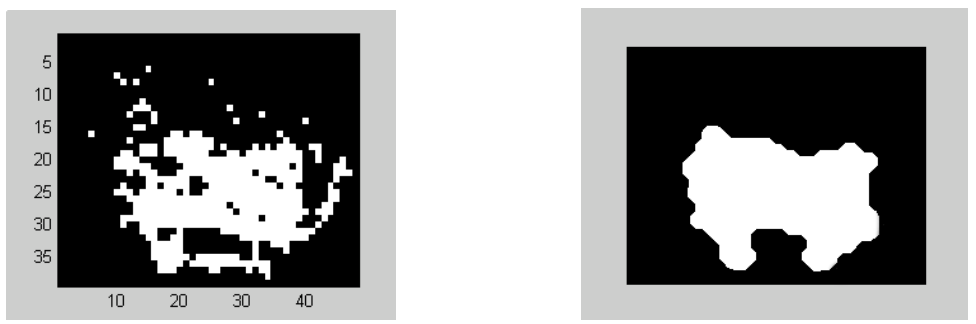

Fig. 3. Binary image produced by SOM clustering (left) and the result of closing followed by opening of the image (right)

\section{Experimental Results}

In this section, we evaluate our algorithm by comparing the algorithm-based segmentations and the manual segmentations on ten US images. The original images are 8bits pixels of size 489x382. These images, however, were resampled by a ratio of 5 after segmentation for speed at the expense of spatial resolution. We asked one expert radiologist to manually segment 9 of the ultrasound images of 3 different individuals. An error analysis on the overlapping area between the segmented areas using the manual and automatic segmentation method is shown in Table 1. In order to calculate the maximal shortest distance error, we find the distance to the closest point on the contour drawn by the expert and we take the maximum of the distances over the contour produced by the algorithm. The overlap area error is the overlap between the manual segmentation and automatic segmentation contours. Our algorithm has demonstrated an accuracy of at least $91 \%$. We believe that the degraded spatial resolution caused by subsampling presents a major source of error that can be drastically reduced if the full image size is considered. The speed of our algorithm has also been tested on a regular $789 \mathrm{MHz}$ desktop PC and recorded an average execution time of 12 seconds.

Table 1. Comparison of the automated and the hand labeled segmentation results

\begin{tabular}{lll}
\hline Individual & Distance (Pixels) & $\begin{array}{l}\text { Overlap Area } \\
\text { Error\% }\end{array}$ \\
\hline 1 & 9 & 2.6 \\
2 & 17 & 8.7 \\
3 & 13 & 5
\end{tabular}

\section{Conclusion}

An automatic segmentation scheme has been presented in this paper, for extracting prostate from TRUS images. The proposed method encodes gray-level features of prostate TRUS images to be used as input to SOM network. While our method does not give high accuracy compared with other contour deformation methods, it requires 
no human intervention at any point in the segmentation process. Future work will focus on a hierarchical strategy that incorporates multiresolution information and improves speed.

\section{References}

1. Zaim, A., Keck, R., Selman, S., Jankun, J. "Three-Dimensional Ultrasound Image Matching System for Photodynamic Therapy", Proceedings of BIOS-SPIE, vol. 4244: 327-337, 2001.

2. Jankun, J., Zaim, A. "An image-guided robotic System for photodynamic Therapy of the Prostate," SPIE Proceeding, vol. 39, pp22-22, 1999.

3. Ghanei, A., Soltanian-Zadeh, H., Ratkesicz, A., Yin, F. "A three-dimensional deformable model for segmentation of human prostate from ultrasound image", Medical Physics, Vol. 28, pp. 2147-2153, 2001.

4. Hu, N., Downey, D., Fenster, A., Ladak, H. "Prostate surface segmentation from 3D ultrasound images", IEEE International Symposium on Biomedical Imaging, pp. 613-616, Washington, D. C., 2002.

5. Pathak, S., Chalana, V., Haynor, D., Kim, Y. "Edge-Guided Boundary Delineation in Protate Ultrasound Images", IEEE Trans. Med. Img., Vol. 19, pp. 1211-1219, 2000.

6. Shen, D., Zhan, Y., Davatzikos, C. "Segmentation Prostate Boundaries from Ultrasound Images Using Statistical Shape Model”, IEEE Trans. On Med. Img., Vol.22, pp. 539-551, Apr.2003.

7. Aarnink, R., Huyanen, A., Giesen, J., De la Rosette, D., Debruyne, F., Wijkstra, H. "Automated prostate volume determination with ultrasonographic imaging," Journal of Urology, vol. 155, pp. 1038-1039, 1996.

8. Castleman, R. Digital Image Processing, Upper Saddle River, New Jersey: Prentice-Hall, 1996.

9. Niblack, W. An Introduction to Digital Image Processing, Massachusetts: Upper Saddle River, New Jersey: Prentice-Hall, 1996.

10. Gonzalez, R. Digital Image Processing, Massachusetts: Addison-Wisely, 1996.

11. Kohonen, T. Self-Organizing Maps. Springer-Verlag, 2001. 